\title{
“Queríamos Cambiar el Mundo, pero el Mundo nos Cambió a Nosotras": Performatividad y Poder Regulador en Protocolos de Género Universitarios
}

\author{
"We Wanted to Change the World, but the World Changed Us": \\ Performativity and Regulatory Power in University Gender \\ Protocols
}

\author{
Claudia Calquín Donoso * \\ Universidad de Santiago de Chile, Chile
}

\begin{abstract}
Este artículo analiza la protocolorización del género en las universidades chilenas como resultado de la institucionalización de las demandas estudiantiles feministas del año 2018. Tomando conceptos de la teoría de la performatividad de Judith Butler, el argumento que se desarrolla es que los protocolos son regulaciones normativas que obedecen a formaciones de poder y versiones hegemónicas de conceptualizar el sujeto mujer, y cuya falta de examinación, supone el riesgo de re naturalizar prácticas y operaciones de exclusión derivadas del régimen político de la heterosexualidad. Asimismo, la protocolorización de la violencia sexual, su objetivación en procedimientos jurídicos y una semántica del hecho, si bien responde a una necesidad de brindar mayor seguridad a 1@s integrantes de la comunidad universitaria bajos criterios consensuados, también corre el riesgo de ser reducida estrictamente a un problema jurídico en que la dimensión más amplia de la violencia como son las relaciones de género y el régimen político de la heterosexualidad, la pregunta por la ética y la responsabilidad colectiva se difuminan bajo una racionalidad de responsabilización individual propia de las lógicas (neo)liberales y que el feminismo ha cuestionado profundamente en las últimas décadas.
\end{abstract}

Descriptores: Protocolos de género; Performatividad; Poder regulador; Régimen heterosexual; Violencia de género.

This article analyzes the protocolization of gender in Chilean universities as a result of the institutionalization of feminist student demands in 2018. Taking concepts from Judith Butler's theory of performativity, the argument that is developed is that protocols are normative regulations that they obey power formations and hegemonic versions of conceptualizing the female subject, and whose lack of examination, entails the risk of re-naturalizing practices and operations of exclusion derived from the political regime of heterosexuality. Likewise, the protocolization of sexual violence, its objectification in legal procedures and the hegemony of a semantics of the fact, although it responds to a need to provide greater security to the members of the university community under consensual criteria, also runs the risk of be strictly reduced to a legal problem in which the broader dimension of violence such as gender relations and the political regime of heterosexuality, the question of ethics and collective responsibility are blurred under a rationale of individual responsibility typical of the (neo) liberal logics that feminism has deeply questioned in recent decades.

Keywords: Gender protocols; Performativity; Regulatory power; Heterosexual regime; Gender violence.

\footnotetext{
*Contacto: claudia.calquin@usach.cl
}

Recibido: 


\section{Introducción}

La aspiración revolucionaria es algo que no tiene oportunidad que desembocar siempre, en el discurso del amo. Ustedes aspiran a un amo, lo tendrán.

(Jaques Lacan, 1968)

Con la expansión de la categoría analítica de performatividad incorporada en el feminismo por Judith Butler a partir del trabajo del lingüista John Austin, investigadoras y pensadoras de diversos campos de las ciencias sociales y las humanidades, han dedicado cada vez más su atención a la manera en cómo u otro tipo de fenómeno o comportamiento acaba siendo conformado como un "problema". En Chile contemporáneo, el problema de la sexualidad juvenil, ha recorrido un largo y espinoso camino desde las mediáticas Jornadas de Conversación sobre Afectividad y Sexualidad -conocidas como JOCAS y la promulgación de la Ley $\mathrm{N}^{\circ} 20.418$ que estableció la obligatoriedad de la educación sexual tras el retorno a la democracia. Durante el año 2018, y de la mano de los movimientos estudiantiles y organizaciones feministas, la preocupación por la sexualidad como problema, se desplazó hacia el sexismo como problema, constatando a la universidad, como un espacio sexuado y generizado, y que no estaba libre del abuso, la exclusión e inclusive, la corrupción. El problema entonces, para las movilizaciones feministas, era menos la sexualidad como si el problema del abuso, la discriminación y el acoso sexual acuñados bajo el nombre genérico de sexismo, fenómeno que insertaba la dimensión ética e inclusive moral de la relación pedagógica.

A pesar de lo promisorio que resultaron las consignas "equidad de género" y "educación no sexista” como locus de enunciación del conglomerado de prácticas críticas del género, su institucionalización y formas de implementación en las universidades rápidamente fueron capturadas por instancias expertas, planificaciones estratégicas, departamentos jurídicos y lógicas institucionales en que pareciera que no todas las voces y debates fueron incorporadas en dicho proceso, cuestión que podría deberse a las desiguales capacidades de negociación de algunos actores y actrices en las instituciones universitarias. Por el contrario, las demandas por la equidad de género y la denuncia de la violencia, se fueron pacificando y estratificando hacia un movimiento de judicialización, en que el horizonte crítico que ofrece la pregunta por la diferencia sexual fue acallado y reificado. Aunque las universidades han invertido esfuerzo y recursos para implementar medidas de equidad y erradicar el sexismo y la violencia, tal como nos alertan Butler y Athanasious (2017) aislar a los sujetos responsables e inclusive repudiar sus acciones, no exime de la necesidad de lograr una explicación e intervención más amplia de la violencia y la discriminación por motivos de género y sexuales, y que pensamos, nos colocaría en un plano de responsabilidad universitaria diferente.

La violencia y la equidad de género lleva a las viejas preguntas sobre ¿cuál es la relación entre cuerpo, identidad y poder?, ¿es posible una resistencia a la violencia de género sin al mismo tiempo afirmar su soberanía? y ¿es posible aproximarnos a una ética feminista de la alteridad sin restaurar una política de la identidad? A partir de estas preguntas, quiero demostrar en estas líneas, que el sexismo y la violencia de género no son residuales a la producción política del género o que pueda ser aislada en la forma de situaciones o hechos de violencia, dado que el género se produce como formación social e historias de subordinación, dominación, despojo y rechazo. Si para Marx, la alienación, es la marca del devenir sujeto en las relaciones de explotación, cabe preguntarse si el sexismo, tiene esa 
misma estructura de marca y si es un indicador de la función alienante de las mismas relaciones sociales del género. Si para Marx esa relación es capitalista para Wittig, esa relación es heterosexual. Desde ese punto de vista, la violencia sexual siempre es una violencia política o una violencia de la política del género, con lo que nos preguntamos acerca de los límites y riesgos de los protocolos de gestión de la violencia si no se examina la misma práctica regulatoria del género o no se reconoce cómo se incorporan y cómo se viven esas regulaciones por parte de los sujetos sobre los cuales se imponen. Si la teoría feminista es teoría crítica, no examinar las propias categorías que los protocolos declaran y constatan, es restaurar una problemática teoría del género que no toma en cuenta las numeras críticas que alertan sobre la violencia que hay tras toda política que asuma una metafísica de la sustancia (Butler, 2016) y sus efectos universalistas de lo femenino y lo heterosexual. Con todo esto, no solo se corre el riesgo de que dichas medidas no sean eficaces, sino, re-naturalizar las normas que producen la exclusión y la violencia.

Considerando que las luchas por la equidad de género, han creado de forma inédita las condiciones para hablar de un sujeto incardinado y que sin lugar a dudas celebramos, el análisis que se ofrece tiene por objetivo examinar los presupuestos ontológicos y normativos que se despliegan en la institucionalización de cierto feminismo de corte jurídico e identitario, que pareciera ser la única posibilidad para la emergencia y reconocimiento de ese sujeto. Quiero demostrar de qué manera las prácticas de protocolorización, las explicaciones de la violencia sexual, las operaciones discursivas que operan en ellos, apuntalan una categoría mujer que no logra desprenderse de sus huellas esencialistas, así como la invisibilización de identidades complejas que no caben dentro de las categorías hombre masculino o mujer femenina; es decir, marcar las ausencias y actos de repetición de normas hegemónicas que, pese a las retóricas del cambio en las que se desenvuelven, no se han modificado sustancialmente. De esta manera, intento aportar a una discusión crítica feminista en que la pregunta por la mujer se desplaza a la pregunta por el poder y de esta forma, asumir los propios ejercicios de exclusión del feminismo, los insistentes velamientos de la versión jurídica de la violencia que al no remover o cuestionar sus bases más profundas, inhiben la potencialidad del género y la sexualidad para transformar las formaciones de poder que se cuestionan en las demandas estudiantiles. En este trabajo, abordaré, los problemas que, desde mi lectura de algunos conceptos de Judith Butler y otras autoras denominadas pos estructuralistas, presenta el discurso de la equidad de género, tal como ha sido institucionalizado en los espacios universitarios, protocolorización, representación, matriz heterosexual y judicialización de la violencia. Una lectura, que siguiendo a Diane Fuss pone el interés en "leer como feminista" las categorías y prácticas que se despliegan con la institucionalización de estas demandas, un leer que nos lleva un ethos de la lectura que busca en las prácticas textuales sus "dobles marcas", es decir su interés por aquello que, en la producción de la verdad ha sido y sigue siendo desterrado.

\section{2. ¿Qué puede un protocolo? Poder regulador y género}

La educación no sexista y la equidad de género son ideales normativos deseables por gran parte de las comunidades educativas, ya que ponen en el centro de atención esferas de la relación pedagógica que poco interés han recibido o han sido negadas en el campo pedagógico y universitario. Se trata del resultado de décadas de prácticas críticas que problematizan ese sujeto, al mismo tiempo que estimulan la pregunta por el deseo, la corporalidad y la identidad en la transmisión de conocimientos; junto con cuestionar las 
relaciones de dominación y exclusión en y de la educación, procuran alternativas epistemológicas y didácticas nacidas de una nueva "imaginación pedagógica" en que el sujeto se complejiza al disolver algunas dicotomías claves de la educación moderna. Pese a la riqueza de estas propuestas, la equidad de género y las demandas impulsadas por las estudiantes adquirieron de forma hegemónica el carácter de implementación de protocolos de actuación, llamados comúnmente protocolos de género-protocolos de lenguaje no sexista o lenguaje inclusivo y protocolos para la prevención del acoso sexual.

Según el Diccionario de la Lengua Española el término protocolo procede del bajo latín protocollum, y éste del bajo griego $\Pi \rho \omega \tau$ con los datos de su autentificación. La entrada de la versión del año 2006 presenta como primera acepción "una regla ceremonial diplomática o palatina establecida por decreto o por costumbre"; en la del 2019, cambia a "serie ordenada de escrituras matrices y otros documentos que un notario o escribano autoriza y custodia con ciertas formalidades". Así, en la última versión del Diccionario, el protocolo aparece como continente más que contenido. Otero (2015) informa que para la Enciclopedia Universal Ilustrada Europeo Americana (Espasa) se trata de una serie de documentos (escrituras matrices, actas, libros, minutarios o escrituras) que recogen textos de importancia y de valor legal. También, que hacia el siglo $\mathrm{V}$ en el imperio Bizantino, protocolo era el libro que los dignatarios llevaban en las ceremonias públicas y que contenían las instrucciones del Emperador para la administración de las provincias. Se trataba de "encuadernados en cuero verde, rojo, azul o amarillo, cerrados con correas o corchetes y adornados de varillas de oro horizontales o formando rombos o losanges, con el retrato del emperador pintado o dorado en las tapas" (p.35) Estos libros serían los verdaderos protocolos o proto kollos, la primeras copia u original. Así protocolo, habría pasado de ser el documento o el libro a las normas referidas al ámbito de actuación del poder contenidas en ellos. Asimismo, protocolo en la actualidad se confunde con ceremonia o aparece como adjetivo -por ejemplo, ceremonia protocolar. Esto nos lleva a lo naturaleza teatral del protocolo a la vez que a su carácter oficial; a esa marca del rostro del emperador en el uso bizantino.

A partir de su etimología e historia, los protocolos exhiben la ambigüedad de aparecer como el poder, el contenedor del poder y podríamos agregar en su uso contemporáneo (ceremonia) un modo de actuación y escenario fabricado para el espectáculo del poder. Así, estas acepciones y usos, hablan sobre el modo en cómo se deben hacer las cosas procedimientos- y al cómo se deben hacer las cosas oficiales, aquellas que llevan la marca del poder. En estos términos, se trata de instaurar un cierto orden oficial de las cosas, a la vez que una escritura que se elabora desde el lugar del poder y que garantiza en su ausencia, su presencia; por lo tanto, se trata de un funcionamiento del poder que lo ubica tanto adentro como afuera del mismo protocolo; es su marca de inscripción, su representante. El protocolo por lo tanto es un texto que con-memora la autoridad del emperador ausente-se usaba en las provincias donde no residía el emperador- y con el cual marca su presencia en tanto rostro y voz; una marca que no es cualquiera sino una escenificación, una ceremonia, una performance que lo instituye como una marca originaria (la primera copia, la copia original, la marca del rostro del rey).

Por otro lado, el protocolo es un vértice en que se encuentran tres formaciones discursivas: penal, moral y de la gestión organizacional. En ese sentido, es una modalidad compleja dado que modela conductas, instituye un modelo de lenguaje, sanciona y establece procedimientos de acceso a la ley, entre otras cuestiones. En esta multiplicidad, su singularidad radica en que, siguiendo a John Austin (1991) a pesar de que están revestidos 
de expresiones descriptivas -en una forma gramatical paradigmática- conllevan "en su misma enunciación una cualidad operativa intrínseca que impone modelos de comportamiento, produce realidades nuevas o modifica las situaciones existentes" (Banderas, 2012, p. 9), es decir, posee manifiestas propiedades performativas.

Como decíamos en párrafos anteriores, los protocolos universitarios rápidamente asumieron el nombre de protocolos de género; por lo que algo del orden de la metonimia se desliza entre protocolo y género y viceversa, junto a algo del orden de procedimientos oficiales y de normas de "cómo hacer el género" en la universidad. Lo interesante, si seguimos al mismo Austin (1991), el protocolo de género nos lleva a la pregunta misma de la naturaleza del género, pues lo que revelan no es una función constatativa del género, sino sus propiedades performativas, en que el género es constituido por actuaciones protocolares, es decir por actuaciones de un poder que adquiere, de acuerdo a Foucault (2006), la forma de un poder regulador.

Si seguimos la clave foucaultiana del poder regulador, advertimos que se trata de una manera de gestión de los cuerpos y las conductas, y que opera a través de la norma. El punto de partida es distinguir la norma de la ley, dado que, a diferencia de la primera, esta instaura prohibiciones con fuerza coercitiva -aunque esto no quiere decir que en la casuística esta distinción no sea arbitraria, o que ley y la norma no se refuercen ni se enreden o que cuando fracasa el poder regulador continúa el poder jurídico, o viceversa-. En el esquema foucaultiano, la norma no establece prohibiciones ni es coercitiva, es productiva y requiere del compromiso o reconocimiento del sujeto, de esta manera, la norma transforma las restricciones y prohibiciones de la ley en un mecanismo transformador del propio sujeto. Con Foucault, vemos que la norma desplaza la concepción jurídica del poder (restricción, prohibición y coerción) a un mecanismo regulador que tiene por fin individualizar, modelar, transformar, etc. al sujeto en el marco de una relación. En es ese sentido, no se trata de un poder externo que ofrece refuerzos y castigos, al modo del conductismo; al igual que el protocolo, la norma es su continente, su contenido y su acción. Macherey (2011) indica que "la norma no obra sobre un contenido que subsista independientemente de ella y fuera de ella, y en sí misma no es independiente de su acción como algo que se desarrolle fuera de ella, en una forma que necesariamente sería la de la partición y la de la escisión” (pp. 108-109). De ahí que, para algunos, el poder regulador trata de un poder a distancia que se apoya en cierta economía de visibilidad que la hace extrapolable a todo el horizonte de lo humano.

Para Francois Edwald (1999), la norma en la propuesta de Foucault trata de "un principio para la comparación, una medida común que se instituye como mera referencia del grupo consigo mismo, cuando el grupo sólo tiene relación consigo mismo, sin una referencia externa y sin verticalidad” (p. 174). La norma es la medida del poder regulador a la vez principio de posibilidad y legitimación de cierta forma de ejercicio político que dispone reticularmente el cuerpo y sus movimientos en el tiempo y el espacio. De esta forma, lo que queda fuera de la norma, no es independientes del espacio normativo, por el contrario, la producción de ese afuera (ya sea en la forma de lo anormal, lo indeseable, etc.) es la medida de su propio funcionamiento; la desviación estándar en la curva normal, marca la misma normalidad. Pensando en este esquema, para Butler (2016) el género es una norma que indica que la relación entre género y poder no es transitiva sino inmanente; es decir, no actúa desde fuera del sujeto ni es anterior a sus mismas acciones lo que impediría la posibilidad de que existiera algo así como una liberación del sujeto de la norma. Esto significa que el género, más que un ideal al cual los sujetos se aproximan, es una forma de 
poder social que produce el campo inteligible de los sujetos, aquello que hace al sujeto legible a los otros, a la vez que un aparato que instituye el género binario (Butler, 2016). Este doble vínculo entre inteligibilidad y diferencia binaria, señala que esta última determina los criterios de inteligibilidad dentro del campo social. En otros términos, instituye una matriz desde la cual las identidades se organizan, los cuerpos se distribuyen y en donde se les otorga un significado específico. Cabe destacar que, para Foucault (2006), lo que se descifra en el poder regulador es el campo siempre móvil y activo de las relaciones de fuerza que producen efectos de estratificación pero que nunca son estables, es "una red de relaciones siempre tensas, siempre en actividad” (p. 83). De ahí que de acuerdo a Butler la norma-es (re) producida a través de su incorporación, "a través de los actos que tratan de aproximarla, a través de las idealizaciones reproducidas en y por esos actos (p.78). Desde este punto de vista, los protocolos de género en tanto, como señalábamos párrafos anteriores, conjunto de actos de habla performativos, se configuran como una performance de género que reproducen la norma a través de sus acciones, sin olvidar que el protocolo implica que la norma es su propia materialidad, su investidura y escenario. A la vez, si el poder regulador es actividad, esto equivale a decir que los protocolos encierran formas múltiples de reproducción de la norma; de este modo, los protocolos son regulaciones especializadas, al punto que el mismo protocolo puede ser objeto de un protocolo anterior que indica cómo ellos mismo deben ser hechos.

En este marco, el problema general del sexismo en los protocolos de género, se atomiza y especifica en una anatomía del poder en cada conducta fuera objeto de una tecnología específica. Así, los protocolos de lenguaje inclusivo y los de acoso sexual fundan prácticas y operaciones diversas -a pesar de que performan el género como algo dado-, por lo que no son reductibles y obedecen a funciones y temporalidades distintas. Los primeros, apuntarían a modelar el lenguaje y la representación en la forma de un (auto)control del acontecimiento discursivo, y el segundo, a una sanción normalizadora (Foucault, 2006) que tendría por fin especificar actos bajo cierto número de categorías generales en la cuales se mueve la oposición binaria de lo permitido y de lo prohibido. Al decir de Foucault, estos últimos establecen una "infra-penalidad" que retícula espacios que las leyes dejan vacío o que todavía no han sido alcanzado por ellas.

\section{Mujeres y lenguaje: Esa espinosa relación entre género y representación}

Los protocolos de lenguaje no sexista han sido definidos como orientaciones que "ofrecen formas para emplear un lenguaje inclusivo, en todo tipo de situaciones y formas de comunicarnos" (Utem, 2019). Generalmente estos abogan por el abandono de un lenguaje basado en el uso universal del género masculino, a través de lo que se llama la duplicación -ya sea en forma completa o abreviada- y/o el uso de sustantivos metonímicos genéricos, en que la diferencia de género se disuelve. A la vez, se incorporan otras recomendaciones, de orden semánticos y pragmáticos, como, por ejemplo, evitar bromas o comentarios basados en estereotipos de género o relativos a conductas sexuales. Así, los protocolos gobiernan todas las dimensiones del lenguaje e incorporan el lenguaje visual y corporal. En algunas universidades, la regulación del lenguaje aparece también en protocolos de prevención del acoso sexual en los que se recomienda, no hacer uso de material pedagógico con imágenes sexuales que denominan explícitas, evitar miradas con connotación sexual, no hacer referencias a las identidades sexuales o comentarios que 
puedan incomodar al estudiante o evitar situaciones o distancias corporales que pueden ser interpretadas como acoso o incomodidad; regulaciones que atienden a las ambigüedades respecto a la posible sexualización de las relaciones pedagógicas.

El lenguaje no sexista y la prevención del acoso sexual -definido como delito en nuestra legislación chilena- se enredan agonística y problemáticamente, en una serie de regulaciones que rebalsan su carácter modelador, para deslizarse a lo que hemos llamado lo infra-penal y viceversa, sugiriendo que la misma naturaleza equívoca del lenguaje, revelaría una dimensión que habría que modelar, controlar y sancionar. Así, el uso de estos particulares verbos y adjetivos como connotación, explícito o incomodar interroga a la interpretación como problema, o lo que podríamos situar en el control de la inherente opacidad del lenguaje como aquello que no está pre-visto. Cabe preguntarse ¿qué inconvenientes trae para la teoría y ética feminista la voluntad de control del discurso?, ¿cuáles son sus efectos?, ¿qué restaura la síntesis disyuntiva entre recomendaciones para un lenguaje no sexista o inclusivo con la prevención y sanción del acoso sexual?, es decir, preguntarse por los efectos de la síntesis confusa entre modelar y sancionar.

La relación entre lenguaje y género es una clave en el feminismo a través de la cual surge la pregunta sobre la producción y reproducción del género como categoría social a la vez que lingüística, indagando con mayor fuerza en el estudio del lenguaje y su función en la reproducción de las versiones normativas del género y el problema de la representación de las mujeres en él; esto ha dado lugar a intensos debates y posicionamientos que apelan a lo que podríamos llamar, la ontología del género y del mismo feminismo-que no equivale a señalar que el género sea solo es una cuestión lingüística. Butler (2016) como Spivak (2009) han resaltado, la doble matriz de significado de la noción de representación y con esto, las ambigüedades y efectos no previstos del uso de las categorías. Por un lado, la representación de las mujeres y sus intereses como base de las consignas feministas, obedece un orden operativo o táctico dentro de un campo de fuerzas políticos. A la vez que sujeto que se intenta desmantelar, es al cual al que se le procura la representación política en las demandas por mayor equidad de género y que ha servido, para introducir los intereses y los objetivos del feminismo en diversos ámbitos en que las desigualdades son incuestionables -salud, educación, justicia, etc.- posibilitando mayores cuotas de igualdad para la mitad de la población -inclusive el hecho de que los cuerpos feminizados puedan discutir la categoría mujer en la universidad. Pero, así como el uso retórico y estratégico de la categoría mujer es utilizado como un reclamo de la identidad, es el origen en el cual se reúnen las exclusiones y silencios abordados por múltiples feminismos que no caben dentro de la corriente principal y revelando las complejas relaciones entre exclusión, violencia y construcción social de las identidades ${ }^{1}$.

Por otro, la representación aparece como función normativa del lenguaje, que de acuerdo a ciertos análisis, como los vinculados al feminismo de la diferencia ${ }^{2}$ implica dar cuenta de las distorsiones o la imposibilidad de acceso a lo que se considera verdadero de las mujeres.

\footnotetext{
${ }^{1}$ El trabajo pionero de Chandra Mohanty, Bajos los ojos de occidente, alerta sobre el problema de la representación de las mujeres de color en el feminismo académico del primer mundo, y la construcción excluyente de la verdad en estos enunciados feministas, en que las mujeres no occidentales son definidas desprovistas de agencia y devaluadas y en relación a una norma liberal. ${ }^{2}$ Véase autoras como Luce Irigaray (El cuerpo a cuerpo con la madre; Espéculo de la otra mujer, entre otros) y los de Luisa Muraro (El orden simbólico de la madre; Tres lecciones sobre la diferencia sexual, entre otros)
} 
Los intereses estratégicos del feminismo, para pensadoras como Luisa Muraro o Luce Irigaray, apuntaría a hacer visibles la verdad de las mujeres que es posible hallar en el cuerpo de la madre, o en el lenguaje materno y con esto, oponer a lo que se designa como el logo-falo-centrismo un lenguaje alternativo que las represente de manera adecuada. Si bien, esta corriente asume la práctica desnaturalizadora de la obra de Simone de Beauvoir, tras la sentencia no se nace mujer, se llega a serlo, este feminismo no ha logrado dar respuesta a la pregunta de cómo sería ese ser en el llegar a ser, o cómo se llega a ser o si hay posibilidades de que los cuerpos signados mujer al nacer puedan llegar a ser otra cosa que no sea, ser mujer. Vemos que a pese a los intentos de la mayor parte de los feminismos de no abandonar totalmente la categoría y reteniendo sus aspectos críticos en lo que ha sido en llamar un esencialismo estratégico (Spivak, 1987) el problema de la representación de las mujeres no puede darse por sentado ni resuelto, sobre todo si el llegar a ser -mujersigue planteando dificultades teóricas y prácticas desde su inicio, y actualmente retorna apasionadamente en los debates sobre la categoría trans ${ }^{3}$.

Tomando en cuenta estos debates, la protocolorización evade la pregunta de si es posible la representación de las mujeres tanto en la categoría mujer, en lo femenino y la duplicación. Desde mi punto de vista, presuponer una respuesta afirmativa sin abordar estas interrogantes trae el riesgo de evadir el problema no resuelto de la misma diferencia sexual y la sentencia beavoriana del llegar a ser. En un primer nivel, se evita el problema del poder del lenguaje para conformar categorías, y en otro, el poder -regulador- en el lenguaje que lo habilita como conformador de categoría. Si el lenguaje es productor de categorías normativas tanto en sus sentencias como en sus ausencias, es porque algo actúa más allá de él. La semiótica destaca que la producción de significados es irreductible a un individuo y al orden lingüístico, pues obedece a estructuras de significados y órdenes más amplios que escapan a la voluntad del sujeto, en tanto matriz de su producción. Esto no quiere decir, que el lenguaje sea una estructura inamovible. Lo que intento establecer es si el lenguaje es productor de categorías, los protocolos no constatan una identidad que ha sido invisibilizada, porque el problema de la representación de las mujeres es justamente su imposibilidad, sino que restauran y desplazan la diferencia binaria a través de otro diagrama de poder, no excluyente sino biopolítico o de control, entendido como régimen de poder específico de una sociedad postdisciplinaria en que el lenguaje es su principio de organización (Deleuze, 1999). Se trata de un diagrama que no sanciona la diferencia, sino más bien la hace visible, la gestiona y gestiona su conflictividad y su potencial subversivo, por medio de la extracción modulante de la propia capacidad simbólica del sujeto. Un poder a distancia, difuso, que ocupa el lenguaje y la comunicación como medios de normalización y dispositivos de (auto) vigilancia.

\section{La matriz heterosexual y el problema de las categorías hombre y mujer en la universidad}

Llegado a este punto, la pregunta que emerge acá es sobre la matriz de ese poder regulador del género de los protocolos universitarios. Si el género no es una sustancia, es una norma

\footnotetext{
${ }^{3}$ Es sintomático, como estos feminismos que defienden la representación de las mujeres también sean las más férreas defensoras de la coherencia sexo-género, a punto en que las identidades trans han debido transitar por nuevos espacios de exclusión y discriminación esta vez, en el mismo feminismo.
} 
y, los protocolos sugieren, que la norma es su propio contenido como su propio escenario de actuación, esto quiere de decir que el género se produce en una matriz y un marco de inteligibilidad específico, en que la normalidad y el principio de inteligibilidad es la coherencia entre sexo, género y deseo. De ahí que la mujer normal, o la mujer como elemento del binario disyuntivo que la duplicación restaura, es la mujer, suficientemente femenina y suficientemente heterosexual. El gran acierto de Butler, a partir de la noción de performatividad es cuestionar esa coherencia y cambiar de dirección esa relación, en que el sexo no sería el punto de partida o base para todo lo demás, sino efecto de la matriz reguladora del deseo. Esta singular relación, Monique Wittg (2002) la denominó matriz heterosexual y Adrienne Rich (2014), heterosexualidad obligatoria. Cabe destacar que esta matriz, no vendría a ocupar el lugar del patriarcado proclamado por un sector del feminismo, ya que en tanto matriz no tiene lugar fijo, ni es un atajo para restaurar una formación universal; no se trata de una estructura que actuaría sobre mujeres y hombres (al no tener lugar no está sobre ni bajo) o dicho de otra forma , no se sustenta en un modelo jurídico en que el sujeto pre existe al poder que se emplaza como relaciones estrictamente disyuntivas (patriarcado v/s mujer; hombre v/s mujer).

Los trabajos de estas autoras, como crítica a las concepciones dominantes y de corriente principal de la relación entre mujeres, lenguaje, representación y política que describimos párrafos anteriores, emergieron tempranamente en el feminismo contemporáneo, mediados del siglo XX, con las prácticas textuales críticas del activismo lesbofeminista, que cabe destacar, dirigieron sus primeras acciones opositivas en dirección a su propia falta de representación en el movimiento de los derechos homosexuales y en el movimiento de las mujeres. El trabajo de Monique Wittig, El pensamiento heterosexual (Straight Mind), publicado originalmente en 1980, es reconocido por impulsar el giro más importante en el feminismo y que remeció sus bases a partir de un gesto problematizante de la afirmación de un "punto de vista de las mujeres" o una "literatura de mujeres". El problema, para Wittig, no es si existe el espacio para un punto de vista de las mujeres; el problema que identifica en este axioma, es si existen las mujeres, y si han de existir, a qué orden obedece esa existencia; se trata de delinear una pregunta crítica que cuestione la misma categoría mujer y su relación de alienación con las experiencias de las mujeres concretas.

El trabajo genealógico que la pensadora francesa adelanta, consiste en deslizar esa falta de representación de las lesbianas en el feminismo, o mejor dicho, esa representación como falta desde una mera ampliación de la categoría mujer que resultaría en las lesbianas también son mujeres, a otro lugar, a un punto cero, en que las lesbianas no son mujeres; las lesbianas son el punto de inflexión de un feminismo que tiene como punto de partido a la mujer, desplazando por lo tanto, la discusión hacia una discusión que el feminismo nunca había pensado: la heterosexualidad. Wittig (2002), al introducir el cuestionamiento de la categoría, avanza a la práctica de categorización misma que crea la diferencia aparentemente natural de hombre y mujer. Este "lesbianismo materialista" que ubica a las mujeres como clase, realiza la heterosexualidad, no como preferencia sexual, sino como régimen político. Demuestra, que la diferencia sexual no es la base de un género, sino resultado de formaciones de poder; no es una división pre existente o fuera de la economía política de la heterosexualidad. En uno de los ensayos que inicia el libro referenciado, señala, 
relación que está en la base de la sociedad (heterosexual), y a través de ella la mitad de la población -las mujeres- es «heterosexualizada» (la fabricación de las mujeres es similar a la fabricación de los eunucos, y a la crianza de esclavos y de animales) y sometida a una economía heterosexual. (Wittig, 2002, p. 26)

Si la economía política de la heterosexualidad es la que crea la categoría mujer, entonces la sentencia "la lesbiana no es mujer" es el resultado lógico del hecho de que mujer solo es concebible dentro del régimen de poder de la heterosexualidad. Más aún, nos dice que la opresión de las lesbianas consiste, precisamente, en colocar a las mujeres fuera del alcance de otras mujeres ya que "las mujeres pertenecen a los hombres" (Wittig, 2002, p.35). Así, la mujer lo es, en tanto lo es para un hombre y una lesbiana debe ser cualquier otra cosa, una no-mujer, un no-hombre. Con este trabajo inaugural de Wittig, la heterosexualidad es algo más que una orientación o una práctica sexual, o mejor dicho la heterosexualidad funciona como la orientación recta de la cual se derivan las desviaciones; es necesario destacar que el vocablo straigh que ha sido traducido como exclusivamente heterosexual, también significa recto.

El giro wittigriano al cuestionar la mente recta-heterosexual condujo a la proliferación de discursos y prácticas desestabilizadoras de la heterosexualidad que se agruparon bajo las etiquetas de lo queer. Más allá de las categorías, lo cierto es que se trata de nuevos horizontes críticos que brindan un análisis más detallado de la relación entre sexo, género y sexualidad y que poca resonancia han tenido en las estrategias de equidad de género universitarias, mostrando que las políticas de género son múltiples y en disputa. Lo que han hecho las instituciones es simplificar la incómoda complejidad del género y la sexualidad, que desde el pensamiento de Wittig y sus herencias, no están fuera -y son resultado del régimen político de la heterosexualidad.

Analicemos el siguiente extracto in extenso del documento Protocolos de actuación frente al acoso sexual en educación técnico profesional. Sugerencias para su elaboración, del Ministerio de Educación de Chile (2019).

Lo que está pasando en nuestro país y también en el mundo no es solamente un
empoderamiento de la mujer ni un cambio cultural, es un cambio de época, una
reestructuración cultural, donde no solamente la voz, sino que la acción de la mujer
señala que llegó el momento de ocupar los espacios de poder, los espacios dentro de la
sociedad civil, los espacios dentro de los liderazgos que hace muchos años vienen
demandando". El Gobierno del Presidente Sebastián Piñera está plenamente
comprometido con una sociedad que respete a las mujeres en todas sus formas y le
entregue las mismas oportunidades a toda la ciudadanía. Por ello, la falta de
herramientas normativas para abordar cabalmente el problema y la ocurrencia de
casos de acoso sexual de interés público plantean urgentemente la necesidad de que, en
su autonomía, las Instituciones tomen medidas para la prevención, atención a las
víctimas y desarrollo de acciones para su erradicación. (p. 7)

Antes de analizar el extracto del protocolo, queremos proponer un experimento textual, que en cada momento en que se enuncia el sujeto se intervenga el texto agregando el sustantivo o adjetivo lesbiana; el texto quedaría de la siguiente forma:

Lo que está pasando en nuestro país y también en el mundo no es solamente un empoderamiento de la lesbiana ni un cambio cultural, es un cambio de época, una reestructuración cultural, donde no solamente la voz, sino que la acción de la lesbiana señala que llegó el momento de ocupar los espacios de poder, los espacios dentro de la sociedad civil, los espacios dentro de los liderazgos que hace muchos años vienen demandando”. El Gobierno del Presidente Sebastián Piñera está plenamente comprometido con una sociedad que respete a las lesbianas en todas sus formas y les entregue las mismas oportunidades a toda la ciudadanía. Por ello, la falta de 
herramientas normativas para abordar cabalmente el problema y la ocurrencia de casos de acoso sexual de interés público plantean urgentemente la necesidad de que, en su autonomía, las Instituciones tomen medidas para la prevención, atención a las víctimas lesbianas y desarrollo de acciones para su erradicación. (p. 7)

Este experimento, puede ser definido como una estrategia de iteración textual y que Butler sitúa en el centro mismo del deshacer el género. Para Butler, develar la matriz performativa del género, supone que el género no tiene un original y se produce como actos repetitivos de una copia que dan la apariencia de sustancia a las categorías hombre/mujer. Lo interesante, es que al cambiar los términos y remplazar mujer por lesbiana se devela, el marco de inteligibilidad que establece la coherencia y sinonimia entre mujer, femenino y heterosexual. Esa repetición, al no ser sobre un original sino sobre una primera copia (proto kollos) nunca es total, y eso permite actos de desplazamientos, parodias, transformación y el propio fracaso de la norma heterosexual. De ahí que el género a la vez que aparato normativo es una comedia, nos dice en El género en disputa (2016), una ficción política, en el sentido de que proporciona posiciones sexuales normativas que son intrínsecamente imposibles de encarnar, y la incapacidad permanente de equipararse plenamente y sin incoherencias con estas posiciones demuestra que la heterosexualidad misma no sólo es una ley obligatoria, sino una comedia inevitable: "En realidad, yo definiría esta idea de la heterosexualidad como un sistema obligatorio y una comedia intrínseca, una parodia permanente de sí misma, y como una perspectiva gay/lésbica diferente (p. 242)

Otra de las autoras que aborda esta matriz, es Sara Ahmed, teórica queer poscolonial. La estrategia desnaturalizadora que Wittig inaugura con la noción de straigh mind, para Ahmed (2019) no necesariamente tiene por efecto desechar la idea de que la heterosexualidad es una orientación como lo hace Wittig. Plantea, que es preciso re significar la noción de orientación y llevarla a sus últimas consecuencias. El giro de Ahmed respecto a Wittig es provocado por la introducción del concepto de orientalismo de Edward Said al feminismo 4 . Del orientalismo, Ahmed avanza hacia las relaciones sexuales pues el orientalismo más que un fenómeno en sí, es un repertorio discursivo, que cruza nuestras epistemologías sobre las relaciones entre el yo y el otro. El yo es cercano e invisible, punto de partida desde el cual se define al otro, es aquello no marcado, el centro ausente respecto al cual los construidos como otros aparecen como exóticos, desviados o como líneas de desviación. Ahmed, también re interpreta el orientalismo gracias a la fenomenología de Husserl -la conciencia está orientada hacia objeto- y la observación de Wittig que la homosexualidad es desviada en tanto el régimen político de la heterosexualidad es lo recto que se erige como la norma desde la cual se miden las orientaciones sexuales. La síntesis que intenta Ahmed, requiere en su formulación crítica, la distinción entre estar orientado hacia un objeto (la conciencia fenomenológica) y estar orientado alrededor de (la conciencia heterosexual/recta).

\footnotetext{
${ }^{4}$ En pocas palabras, y a riesgo de simplificar el concepto, el orientalismo es la creación imaginaria de la categoría oriental y oriente, por parte de escritores y estudiosos europeos, situando a oriente en un espacio marcado como un otro. Lo que Said denuncia por medio del orientalismo es el artificio que hay en la diferencia abismante entre Europa y aquello que no es Europa, de esta forma se trata de una relación política en que la diferencia es disyuntiva y negativa; lo importante es que esa diferencia es una operación performativa, en tanto occidente y oriente no existen como identidades anteriores al proceso de diferenciación; es decir la diferenciación inaugura la diferencia.
} 
Si bien toda conciencia se orienta a su exterioridad, estar orientado alrededor de una cosa remite a la pregunta, según su análisis, de cómo son dispuestos y cómo se hacen coherentes los cuerpos. Estar alrededor implica ser tomado por una cosa, hacer de eso lo central, en definitiva mantener un centro. Si la heterosexualidad, es un estar alrededor de ella y por tanto, norma desde la cual se definen el yo y el otro, quiere decir que esos otros son existentes siempre y cuando le permita a la norma su propia reproducción y legitimación; el otro en tanto otro no tiene posibilidades de existencia a pesar de que esos otros sean integrados; esto muestra que ese "a pesar", esa incorporación del otro no heterosexual no significa una modificación del repertorio y las prácticas excluyentes de la heterosexualidad, como centro ausente, sino su condición misma, en tanto esa misma acción de integrar al otro, es la que mantiene y actualiza la distancia excluyente como fenómeno necesario a la vez que refuerza la cadena de repeticiones paródicas del hacer "como" heterosexual. Cabe destacar que para Ahmed no toda gestión de la distancia, en definitiva, de la diferencia, deba ser dicotómica, es la heterosexualidad la que sitúa los objetos como rectos o desviados; pueden existir gestiones que atiendan a una ampliación de la identidad hacia nuevas capacidades y direcciones, en que el no-yo es incorporado en el cuerpo ampliando el alcance del yo. De esta forma, muy en sintonía con la idea de una "straight mind" en Wittig, la heterosexualidad para Ahmed es una forma de mirar el mundo, es decir una forma de reunir cosas alrededor, de manera que miran en una determinada dirección.

Pese a que la heterosexualidad es una orientación más y una comedia o un drama, los protocolos asumen que las orientaciones sexuales son las otras orientaciones, y a las cuales las universidades deberían dar cabida, gestionar al modo de un teatro de variedades mientras que la heterosexualidad se mantiene invisible. A partir de estas autoras, podemos decir que esa visión institucional no asume que el reglamento del género se realiza por prácticas repetitivas de exclusión, clasificación, ordenación, etc. a nivel de las prácticas y a nivel de los saberes universitarios, que en las políticas de equidad de género permanecen intocables, al punto que por ejemplo, se sigue asumiendo en las mallas curriculares el discurso psiquiátrico de la homosexualidad como patología, o que la misma heterosexualidad no se interroga como sexualidad igualmente construida o como decíamos anteriormente, como comedia siempre al borde de su propio fracaso. Entonces, ¿por qué si se deshace la condición de exclusión de las mujeres resultado de las relaciones de género, no se habla en los mismos términos de las relaciones heterosexuales? , ¿qué hace que dentro del campo de posibilidades de la equidad de género sea el punto de vista de las mujeres heterosexuales el reconocido, y no el punto de vista de las lesbianas? ¿por qué no se eligió una estrategia de lesbianización o queerización de la educación superior?, ¿qué hace que el presupuesto de heterosexual, en el texto del ministerio de educación intervenido más arriba, sea condición de enunciación de las mujeres en la equidad de género?

Butler indica que la elección de la heterosexualidad no solo exige el rechazo al objeto de deseo (el objeto del mismo sexo) sino que el rechazo al mismo deseo en tanto potencialidad. La heterosexualidad es obligatoria porque se expresa como un "nunca jamás" -he deseado a alguien de mí mismo sexo- a la vez que "nunca jamás" desearé a alguien de mí mismo sexo. En ese sentido, la heterosexualidad en tanto obligatoria se funda en una operación de forclusión que debe ser actualizada constantemente - por la razón o la fuerza- y en que la violencia que emerge desde el mismo acto de forclusión, adquiere una función central. De ahí que la violencia de género no puede ser desligada de las historias de la violencia en 
la escuela, del insulto a las minorías sexuales en todo su ciclo vital, entre otras manifestaciones. Como nos dice Butler, en Mecanismos psíquicos del poder, el problema de la violencia es preciso ubicarla en la misma condición de posibilidad del sujeto, pues, así como nos aprisiona, nos daña y estratifica nuestro deseo en un marco binario y disyuntivo, nos proporciona las mismas condiciones de lo que somos. Por ejemplo, las vidas trans revelan como el nombre propio, a la vez que lugar de reconocimiento del yo, es fuente de despojo, lugar de desprecio a la misma experiencia de no coherencia sexogénero. En ese sentido, no hay sujetos que han sufrido violencia, sino más bien somos asujetados por la violencia disciplinaria del aparato regulador de la heterosexualidad.

\section{La voz pasiva y el reclamo del standpoint minoritario}

El análisis de la dimensión pragmática de los protocolos, indica que su contenido propositivo no puede desvincularse del contexto, como nos dice Austin, sino que también "del uso que él hace, en vista de un cierto efecto que se puede producir en el oyente, y todas sus consecuencias prácticas de las cuales él es el responsable por el mero hecho de afirmar" (Chauviré, 2010, p.105). En los protocolos, apreciamos la presencia de una voz particularmente prescriptiva, directiva a la vez que pasiva, que, junto con sancionar, ordenar y establecer conductas, esconde los signos indexicales del lenguaje entendidos como huellas o rastros del sujeto de la enunciación. Para el feminismo, el uso de la voz pasiva tiene un estatus ambiguo, dado que para algunas pensadoras como Dona Haraway o Evelyn Fox Keller, se trata de un estrategia discursiva que oculta el yo generizado que habita la ciencia en tanto empresa humana y práctica social y sexual; a la vez que reviste el lenguaje prescriptivo de la moral con la apariencia de necesidad al situarse desde una ausencia de un punto de vista y que ha sido fuertemente contrastado por los conceptos de conocimiento situado o conocimientos incardinados, entre otros. La voz pasiva, nos lleva a esa voz que habla desde ningún lugar y desde todas las posiciones de acuerdo a Haraway (2004), una voz que imita, para esta autora, la voz del Dios cristiano -que no tienen origen ni final. Pero, también, la voz pasiva para Wittig (2002) es la manera de superar la diferencia de género. Desde esta perspectiva, la polisemia de la función de la voz pasiva, indica que sus multiformes efectos de poder dependen de la posición-sujeto desde la cual se despliega. Esa voz que prescribe, dirige, ordena y sanciona ¿es una voz que está fuera de la diferencia sexual o está más allá del género al modo de un discurso del amo, total y no marcado por la diferencia? Los protocolos, como un conjunto de actos de habla tienen la función discursiva de ser una autoridad invisible. Esa operación de tachadura y sus efectos de no original-idad, es una operación de naturalización que es muy peligrosa para los propios reclamos del feminismo como política localizada y desnaturalizadora al presentar a la norma y a la ley más allá de la diferencia sexual. Derrida (2017) nos dice en su análisis de la parábola Ante la ley de Kafka que entrar en relación con la ley, de la verdad o de la corrección ética y política,

$$
\begin{aligned}
& \text { [...]con eso que dice «Tú debes» y «Tú no debes», es actuar como si ella no tendría } \\
& \text { historia o, en todo caso, como si ella ya no dependería de una presentación histórica y, } \\
& \text { al mismo tiempo, es dejarse fascinar, provocar, apostrofar por la historia de esta no- } \\
& \text { historia. Es dejarse tentar por lo imposible: una teoría del origen de la ley y, por lo } \\
& \text { tanto, de su no-origen. (p.22) }
\end{aligned}
$$

Con esto surge otra interrogante, ¿cuál es la historia de esa voz?, ¿a quién corresponde?, ¿a la minoría o la institución? y si es de la institución ¿de dónde extrae su legitimidad para actuar como ventrílocua de la voz de la minoría?, ¿qué pasa con la capacidad de la minoría 
de hablar por si misma si la institución es su ventrílocua? y si es la voz de la minoría, ¿qué pasa con lo minoritario y su fuerza destructora o de-constructora, cuando ocupa el lugar de la mayoría en tanto voz legitima -el rostro del rey más allá de la diferencia sexualpara gobernar y producir parámetros y medidas de conducta? Estas preguntas lejos de tener una respuesta unívoca, se dirigen al centro mismo de la ética y siguiendo al Foucault de crítica y aufklärung, nos conduce al problema mismo de la universidad como criatura de la modernidad ilustrada. De ahí que la actitud crítica de la ética feminista revela siempre a su contrapregunta: ¿queremos ser gobernadas de esa forma y por esos procedimientos?

Cabe aclarar que estas preguntas se alejan de cualquier intento de simpatizar con esas voces también mayoritarias -cuerpo académico masculino- que hablaron de una nueva cacería de brujas, no solo porque las sanciones no se comparan a la quema en la hoguera sino porque quienes lo enuncian son justamente los cuerpos que nunca habrían podido ser considerados brujas. Mi punto de partida, para intentar una aproximación a las respuestas a dichas preguntas es otro, uno que asume que el lenguaje siempre está regulado por la naturaleza de las instituciones, al punto que se puede hablar de géneros discursivos o lenguaje científico, lenguaje profesional, lenguaje informal, etc. cada uno con sus reglas sintácticas y semánticas que indican que en una disciplina o espacio social no se puede hablar de cualquier forma ni de cualquier cosa ni desde cualquier lugar. La pregunta por la minoría, es hacer explicita la inquietud del feminismo por examinar constantemente y en todos los lugares, la forma que adquiere la representación de las voces feministas y minoritarias y sus efectos performativos para la política del género; es peguntarse si esa voz se estructura como una voz más en el coro de la diversidad multicultural del neoliberalismo, o por el contrario, como una política radical que asume la forma de un standpoint feminista y minoritario, en que no solo quepa hablar por sí mismas, sino que ese hablar por sí mismo, sea una práctica desnaturalizadora y desestabilizadora de las operaciones de poder.

Lo que la pedagoga argentina valeria flores (2017) enuncia como el hablar contra sí misma o la erótica del insulto de Didier Eribo (2004), son tropologías que indican el potencial subversivo de resignificar el insulto y la discriminación como estrategias de conformación de una voz propia en la forma de -permítame un trabalenguas- un standpoint desnaturalizador del standpoint que niega su condición de standpoint -en la huella de Haraway al decir "la cultura de la no cultura"- y con el cual se ha construido la pedagogía moderna y las instituciones universitarias y sus metáforas de claridad, no contaminación y transparencia. A la vez que rechazar las propuestas también críticas de corte marxista, que establecen relaciones estrictamente transitivas entre sujeto y escuela, en que el sujeto queda subsumido y paralizado en la estructura. Desde mi perspectiva, estas propuestas, asumen el feminismo en la educación no como una teoría que habla de las mujeres, o de cuestiones que pasan entre hombres y mujeres; se trata de una lectura y definición del feminismo que podríamos llamar, una metodología con efectos ontológicos que traslada la preocupación por la soberanía del sujeto, a la pregunta por el poder regulador y qué puede ese poder regulador. La fórmula es afirmar el punto de vista de la minoría como un yo que deviene máquina desnaturalizadora y desterritorializadora de las formaciones de la mayoría, en tanto se introducen en dominios claves como la producción del saber universitario, que por regla general se ha definido política e intelectualmente rechazando a las minorías, al usurpar sus propias capacidades de (auto) significación y (auto) representación (Said, 2005). 
Desde este punto de vista, la pregunta sobre si queremos ser gobernadas de esa forma es co-sustancial a la pregunta de si ¿pueden hablar los sujetos minoritarios? y a la vez a la pregunta normativa de como tendrían que hablar los sujetos minoritarios: ¿desde qué lugar hablan?, ¿hablan desde el lugar de lo minoritario o desde el lugar de la instituciónrostro del rey?; y si hablan desde el lugar de la institución y con los mismos recursos y prácticas de la institución ¿pasan a ocupar el lugar de la institución? -iha muerto el rey viva el rey!- o son posibles ejercicios de traducción y desestabilización?, ¿qué sucede con la razón del feminismo, supuesto lugar de representación del sujeto minoritario en tanto potencia alteradora, creadora y apertura a lo nuevo? Si bien estas preguntas recuerdan la gran paradoja, para los movimientos minoritarios y que inspira el título de este trabajo "quisimos cambiar el mundo y el mundo nos cambió a nosotros"- quiero situar estas preguntas en una cuestión más concreta, pero no menos importante, es que esa voz se juega en un contexto en que son muy pocos los protocolos que declaran la perspectiva de género o feminista. En la mayoría de ellos, las demandas feministas fueron capturadas en un proceso de traducción hacia un vocabulario jurídico y de la gestión organizacional, que asegura cierto rasgo de credibilidad, neutralidad y naturalidad de la norma. Una lectura de las ausencias textuales, clave para abordar esa fenomenología de la voz de la autoridad protocolizada, destaca que especialmente en las universidades pontificias las palabras género, poder, homosexualidad, normalización, heteronorma, lesbianismo, queer, homofobia, heterosexualidad, etc. propias del vocabulario feminista estudiantil desaparecen. En otras palabras, el género como problema se soluciona forcluyendo el género como categoría, función del lenguaje, matriz analítica y standpoint, es decir, negando el poder en el lenguaje que habita y lo habilita para conformar o deshacer las categorías, al mismo tiempo, su inexorable función habilitadora de la subjetividad y la capacidad reflexiva del sujeto, como relación consigo mismo; es decir, las operaciones tropológicas del mismo lenguaje para volverse hacia sí mismo.

\section{Crítica a la versión jurídica de la violencia. Del hecho al acontecimiento}

El rodeo por las categorías fundacionales del sexo, género y orientación sexual es un movimiento que me sirve para llegar al punto más crítico de la protocolorización de la violencia y el género. Si en los párrafos anteriores, señalamos que las políticas de equidad de género han evadido la pregunta por la heterosexualidad como régimen político y la heterosexualidad como un acto de violencia, el argumento que expongo a continuación es que los protocolos contra el acoso sexual, realizan operaciones del poder que proscribe el goce, pero como toda prescripción, no lo anula, sino regula sus formaciones y modos de circulación performando de forma indirecta, a la prescripción como promesa de equidad. Esto plantea otra dimensión de la analítica del poder: ¿cuál es la versión, forma o modalidad que adquiere la violencia en los protocolos?

Analicemos un caso, la Política de prevención y apoyo a víctimas de violencia sexual en contextos universitarios de la Universidad Católica. Quizás se nos reproche lo desafortunado del ejemplo, pues se trataría de una institución pontificia que sabemos ha rechazado el concepto de género desde su misma introducción en la Convención de Beijing, tal como lo documenta detalladamente la historiadora Joan Wallach Scott en el prefacio a la edición revisada en inglés del libro Género e historia. Si bien es un caso extremo que no representa a la totalidad de las universidades, no hay que olvidar que esta 
Universidad se considera la segunda más importante en el país y que recibe en esa misma proporción los recursos estatales, entre otros aspectos que interrogan sobre la separación iglesia-estado. Haciendo esta aclaración, en este protocolo la narrativa de la violencia apunta a que la violencia es un hecho de violencia. La violencia como hecho reúne una serie de presupuestos: la violencia sería un estado de cosas transparente, que trataría de conductas objetivas; sería objetivable en tanto se le aparece a un sujeto conformado anterior a las mismas operaciones de la violencia; y sería provocado por un agente que coincide con nuestra idea de responsabilización del marco liberal, o con la del sujeto abyecto del marco moral.

Dicho así, llama la atención la tachadura radical del problema del género y de la misma sexualidad en la violencia sexual; el análisis cuantitativo de la política arroja cero veces la palabra género y la misma violencia se denomina y como decíamos "hechos de violencia sexual". Esta radicalidad de la ausencia de categorías políticas como el género, evade la posibilidad de preguntarnos sobre qué debe hacerse políticamente con el daño y la vulnerabilidad, más allá de proclamar la ley heterosexual del derecho occidental, y nos hace preguntarnos acerca de si la violencia es prevenible, o dicho de otra forma si hay algo de inevitable de la violencia en las relaciones de género. El lugar ausente del género en la cadena significante, y la semántica del "hecho" con usos pragmáticos, intenta cubrir de facticidad y neutralidad el problema político de la violencia, lo que sugiere que la violencia no sería parte de la vida política o es independiente del proceso de la producción relacional del sujeto, desmentido en el análisis de la heterosexualidad. Si bien, siguiendo a Butler (2017), este marco jurídico puede asegurarnos un espacio de protección legal, eso no quiere decir que sea una descripción adecuada de la violencia sexual. En ese sentido, asegurar la posibilidad de pre-venir de la violencia, implica asegurar en qué orden de cosas se define la violencia. Para Butler (2017), la violencia es una dimensión de la vida política, pues nos hace preguntarnos sobre qué "es lo que cuenta como humano, las vidas que cuentan como vidas y, finalmente, lo que hace que una vida valga la pena” (p.46). Esto significa, que la vida, nuestras vidas, se constituyen políticamente en virtud de la vulnerabilidad del cuerpo como lugar de deseo a la vez que dependencia al otro, y lugar público y político de afirmación y de exposición. La violencia nos conduce al problema mismo de la sujeción, descrito anteriormente, a partir de esa disposición de estar dirigido y orientados hacia los otros, a la vez que asujetados y expuestos a otros.

Definir la violencia como un hecho, sugiere fundar un sujeto al margen de su existencia política -una paradoja; politicidad que es condición primaria de la existencia social del sujeto a la vez que cobertura que lo protege de la lucha de todos contra todos del estado natural y de la violencia que lo funda como sujeto. En Vidas precarias Butler nos dice que la vulnerabilidad, la violencia y el duelo son los puntos de partida de la política; la política en ese sentido no es propiedad y esencia del sujeto, la política apela a su exterioridad, a su relación con lo otro, a lo que sucede entre los sujetos a la vez que lo fundan (Arendt, 2019). De ahí que los feminismos han apuntalado su crítica, al imperativo de re politizar la violencia, lo que implica abandonar la violencia como hecho y conceptualizarla como algo distinto. Desde mi punto de vista, la violencia emerge como acontecimiento, no tanto en el sentido de extraordinario, pues hemos definido la violencia como constitutiva; acontecimiento en su aspecto singular y en su relación con un orden de significación. Hecho, es una categoría que corresponde a la epistemología positivista, de un estado de cosas que son independientes del observador; los hechos son autoevidentes corresponden a un orden natural y absoluto; por el contrario, el acontecimiento, como instancia singular, 
se inscribe en un dispositivo de la verdad que reconoce estrategias y juegos de poder (Foucault, 2013); por lo tanto aflora como un terreno intenso de fuerzas y contrafuerzas en que la mirada del observador -el Otro del otro- es constituyente de su propia aparición. En ese sentido, si el hecho es (im) posible de describir, el acontecimiento es preciso de interpretar. Para Hannah Arendt (2019) filósofa de la violencia y el totalitarismo, el nazismo como acontecimiento es la que posibilita pregunta sobre la responsabilidad como dimensión de lo político. Nos dice que pensar el campo de concentración como fenómenos dramáticos de la violencia, es situarlo como un acontecimiento, algo que sobreviene en el tiempo humano; un tiempo plegado al sentido. El acontecimiento revela lo imprevisto y la fragilidad de la acción y de "las palabras que vinculan los individuos entre sî" (Birulés, p.28). De esta forma, su modo de contemplarlo y experimentarlo se define por su dependencia de los procesos que necesariamente interrumpe. Así, el acontecimiento involucra en su aparecer la respuesta del colectivo y su capacidad para con-memorar; podríamos decir que a diferencia del hecho el acontecimiento es una interpelación al Otro de lo otro, es decir, nosotros.

Si somos interpelados por la violencia, Arendt se aproxima a una metodología, en el que interesa alejarse del sine ire et studio ${ }^{5}$, que la alejaría de la objetividad para afirmar el campo relacional de lo afectivo. La violencia no puede ser abordado desde un lugar de objetividad y neutralidad y desde mi perspectiva, desprovista de las categorías políticas que la han denunciado colericámente, pues en sus términos "escribir sin la cólera sería eliminar del fenómeno una parte de su naturaleza, una de sus cualidades inherentes (Birulés, p. 30). La emoción, que para Arendt no es el revés de lo racional, sino el revés de insensibilidad o el sentimentalismo, es lo que nos lleva a la pregunta por la responsabilidad de la violencia, en tanto interpela nuestra capacidad de ser afectados y afectarnos por ella. La violencia como acontecimiento es inseparable -me permito un neologismo- de lo afectual a la vez que de una actitud crítica en que su comprensión nunca es total, por lo que las acciones de protección siempre deben ser examinadas. Si la violencia, nos lleva a la responsabilidad del ser afectado por el sufrimiento ajeno y la política, es aquello que ocurre en el entre los seres humanos, entonces hablamos que la violencia es una categoría política, no un problema de un sujeto abyecto. Acá es clave la distinción entre responsabilización y responsabilidad. Los protocolos apelan a la responsabilización individual y no a la responsabilidad política. Con esto, no sugiero una visión saturada de nihilismo en que pareciera que no hay posibilidades de intervenir en la violencia ni intentar otras modos de relación más equitativas, sino indicar que inclusive las versiones más equitativas e igualitarias de las relaciones de género, no están fuera de las mismas condiciones que generan la violencia, y denunciar siempre aquello, sustenta el potencial de la crítica feminista que nos ha ofrecido diagramas de la violencia de género que no es problema individual, monstruosidad o patología, que la examina en sus dimensiones incómodas y la resitúa en el campo de la ética, la relación y la responsabilidad social.

\footnotetext{
${ }^{5}$ Hay que situar esta "metodología” o el camino que traza Arendt para el análisis de la violencia, a partir de la polémica bien conocida que tuvo lugar, entre ella y Eric Voegelin, a raíz de la publicación en 1951 de Los orígenes del totalitarismo. Este último le reprocha que no llevó a cabo un análisis científico y objetivo de lo acontecido, sino que se enhebraban simplemente una serie de asociaciones metafísicas y le recomienda que se acerque al fenómeno sine ira et studio. Para los detalles de este debate, véase el artículo de Josefina Birulés (2003) Memoria, inmortalidad e historia en Hannah Arendt, Diótima, 1
} 


\section{Agradecimientos}

Este trabajo ha sido realizado en el marco del proyecto "Discursos de la sexualidad y el género en la formación de estudiantes de psicología de la Usach" financiado por el Fondo Proyectos de Innovación Docente (PID) de la Vicerrectoría Académica de la Universidad de Santiago de Chile.

\section{Referencias}

Ahmed, S. (2019). Fenomenología queer. Orientaciones, objetos, otros. Bellaterra.

Arendt, H. (2019) ¿Qué es la política? Ariel.

Birulés, J. (2019). Introducción ¿Por qué debe haber alguién y no nadie? En Arendt, H. ¿Qué es la política? (pp. 9-35). Ariel.

Butler, J. (2015). Mecanismos psíquicos del poder. Cátedra.

Butler, J. (2016). El género en disputa. El feminismo y la subversión de la identidad. Paidos.

Butler, J. y Athanasiou, A. (2017). Desposesión: lo performativo en lo político. Eterna Cadencia.

Chauviré, C. (2010). Indexicalidad y aserción en Peirce. Revista Colombiana de Filosofía de la Ciencia, $\mathrm{X}(20-21), 103-117$.

Deleuze, G. (1999). “Posdata sobre las sociedades de control”. En Ferrer, C (Comp), El lenguaje libertario. Antología del pensamiento anarquista contemporáneo (pp.113-121). Altamira.

Derrida, J. (2017). Prejuzgados. Ante la ley. Averiganes.

Edwald, F. (1999). Un poder sin un afuera. En: Balbier, E., Deleuze, G., Dreyfus, H. y otros. Michel Foucault, filósofo (167-178). Gedisa.

Eribo, D. (2004). Insult and the Making of the Gay Self. University Press.

Flores, V. (2017). Tropismos de la disidencia. Palinodia.

Foucault, M. (2006). Vigilar y castigar. Nacimiento de la prisión. S. XXI.

Foucault, M. (2013). El orden del discurso. Tusquet.

Haraway, D. (2004). Testigo_modesto@segundo_milenio.HombreHembra(c)_conoce_oncoratón( $r)$. Feminismo y tecnociencia. UOC.

Macherey, P. (2011). De Canguilhem a Foucault: la fuerza de las normas. Amorrurtu..

Ministerio de Educación de Chile. (2019). Protocolos de actuación frente al acoso sexual en educación técnico profesional. Sugerencias para su elaboración. https://bibliotecadigital.mineduc.cl/handle/20.500.12365/14489

Otero, M. T. (2015). Historia del protocolo. UOC.

Rich, A. (1980). Compulsory Heterosexuality and Lesbian Existence. Onlywomen Press. https://doi.org/10.1086/493756

Said, E. (2005). Repensar el orientalismo en Reflexiones sobre el exilio. Ensayos literarios y culturales. Debate.

Spivak, G. (1987). In Other Worlds. Essays in Cultural Politics. Methuen.

Spivak, G. (2009). ¿Pueden hablar los subalternos? MACBA. 
Universidad Católica de Chile. (2017). Política de prevención y apoyo a víctimas de violencia sexual en contextos universitarios de la Universidad Católica. https://www.uc.cl/noticias/la-politica-deprevencion-de-agresiones-sexuales-en-el-contexto-universitario-uc/prevencion-y-apoyoa-victimas-de-violencia-sexual-en-contextos-universitarios

Universidad Tecnológica Metropolitana-Utem. (2019). Recomendaciones para uso de lenguaje inclusivo $y$ no sexista. https://www.utem.cl/universidad/genero-yequidad/recomendaciones-para-uso-de-lenguaje-inclusivo-y-no-sexista/

Wittig, M. (2002). El pensamiento heterosexual y otros ensayos. Egales.

\section{Breve CV de la autora}

\section{Claudia Calquín Donoso}

Máster en estudios de mujeres, género y ciudadanía del Instituto interuniversitario de estudios de mujeres y género (IIEDG) de Barcelona. Doctora en ciudadanía y derechos humanos de la Universidad de Barcelona. Profesora asistente de la Escuela de psicología de la Universidad de Santiago de Chile. Investigadora especializada en temáticas de género, feminismo y teoría queer. Ha publicado artículos, capítulos de libros y libros en estos temas. ORCID ID: https://orcid.org/OOOO-0002-4420-5927. Email claudia.calquin@usach.cl 